\title{
Performance Measurement Methodologies and Quality of Service Evaluation in VoIP and Desktop Videoconferencing Networks
}

\author{
Henri TOBIET $^{1}$ and Pascal LORENZ ${ }^{2}$ \\ ${ }^{1}$ NMG TELECOMS - Network Management Group - Telecoms Solutions \\ 20e, rue Salomon Grumbach, BP 2087, 68059 Mulhouse Cedex, France \\ h.tobietenmg.fr \\ ${ }^{2}$ University of Colmar - IUT / GTR \\ 34 rue du Grillenbreit - 68008 Colmar, France \\ lorenz@colmar.uha.fr
}

\begin{abstract}
The present paper relates to performance and quality of service evaluation for VoIP (Voice over IP) and desktop videoconferencing services in IP networks. Simulation-based performance measurements consist in the generation of performance statistics obtained by measurements realized by simulation on the subscriber interface. They include detailed measurement of call quality, call set-up quality and availability. The tests are accomplished by emission of non-disturbing additional test traffic. The real-time QoS monitoring is based on non-intrusive analysis of real call parameters. The advantage of this method is the exhaustiveness of the analyzed call. The QoS measurements are mainly service availability and call set-up quality.
\end{abstract}

\section{Introduction}

QoS defined in CCITT Recommendation E.800 may be considered as the generic definition reproduced below: "The collective effect of service performance which determine the degree of satisfaction of a user of the service".

Quality of Service on the LAN represents a major challenge, not so much during the predictable processes of compressing the voice streams and splitting them into packets which are mathematically predictable, the real challenge is of sharing the connectionless transmission media with other users in a predictable and quantifiable way. As soon as voice/video traffic reaches the IP network, it must compete with electronic mail traffic, database applications and file transfers [13], [17], [18].

QoS evaluation methodologies are based on the previous studies performed in European Projects, such as QOSMIC (QoS Methodologies and tools for Integrated Communications). The resulting QoS will be dependent on the performance of the physical, AAL (ATM Adaptation Layer) and transport layers involved in the protocol stack implementation [1], [10].

Work is currently on-going in modelization of the TCP/IP protocol layer, in terms of performance aspects (delays, etc...). The resulting model will then be applied to 
convert network layer performance (ATM, AAL) to transport layer performance, according to the selected services: telephony over IP, file transfer over IP and videoconferencing over IP [12], [14].

\subsection{QoS Characterization}

The used TCP/IP model can be described as follows:

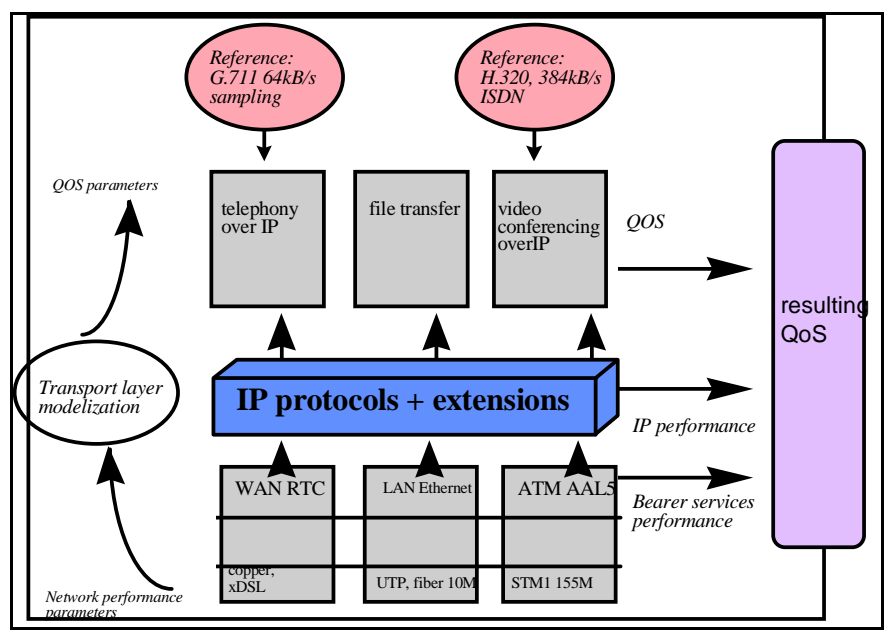

Fig. 1 : QoS protocol oriented approach

The main performance parameter to be measured is the Round Trip Time (RTT) but the performance evaluation will also concentrate on the following aspects: connections opening and closing mechanisms, data transfer mechanisms, addressing, parameters negotiation, congestion control and errors control.

Part of our studies concentrated on end-to-end QoS characterization. End-to-end QoS in a videoconferencing system is characterized under two broad headings:

- call set-up quality and

- call quality.

Call set-up quality is mainly characterized by the call set up time, i.e. the time elapsed from the end of the user interface command by the caller (keypad dialing, email alias typing, etc) to the receipt by the caller of a meaningful tone. ITU-T Recommendation E.600 provides more information on the definition of post dialing delay. Call set-up time is perceived by the user as the responsiveness of the service. Other factors such as ease of use contribute also to the user experience. The first of these factors is objective, the second is subjective [16].

Within the broad category of call quality two major factors contribute to the overall QoS experience of the user of the videoconferencing system:

- the end-to-end delay which impacts the interactivity of a conversation and

- the end-to-end video and speech quality. 
The following factors contribute to the overall call-set up time:

- IP access network set up delays (these would include transport layer set uptimes, modem training times and log on times at the ISP Gateway),

- signaling delays across the IP backbone,

- call set-up delays within the gatekeeper(s),

- access times and call processing delays to back-end services, such as directory services or authentication services,

- call set-up delays within the gateway,

- call set up times in the network(s).

The end-to-end delays are influenced by IP terminal buffering delays, H.323 packetization/buffering delays, codec delays and network transmission/propagation delays. The end-to-end audio/video quality depends on input and output devices, analogue/digital and digital/analogue circuit noise, video and audio coding distortion, effects of bandwidth limitation in the IP network.

The QoS issues associated with the IP terminal are the choice of codecs used in the terminal, the performance of the codec to various types of network degradation, the signal processing delays, the call processing delays, the number of frames per packet, the processing delays associated with security issues, the design of jitter buffers, the delays through the audio or digital media paths and the performance of echocanceling devices [7], [8], [11].

\subsection{QoS Issues Associated with LAN Access}

In this configuration the access layer is limited to the Network Interface Card (NIC) used within the IP terminal. Though the LAN has ample bandwidth for transmission of coded speech/video, a fundamental issue frequently encountered is contention for shared media.

At any time, other (non audio) endpoints on the LAN, may flood the LAN and consume all the available bandwidth. This problem can only be avoided if there are mechanisms to manage and police the use of bandwidth (both for real-time use and best-effort use). The Subnet Bandwidth Manager (SBM) and RSVP (IETF RFC 2205) are intended to provide this capability.

The factors affecting QoS in this scenario are the transmission delays through NIC and the jitter in data buffers associated with the NIC. It is anticipated that these parameters will in general be well controlled and specification of upper bounds on these parameters should present few difficulties.

\subsection{QoS Associated with PSTN Modem Accesses}

In this type of access, modems are used to establish a digital channel between the videoconferencing terminal and the IP network. The factors affecting QoS in this configuration are the:

- modem bit rate,

- modem transmission overheads, 
- throughput delay in modem and at ISP site,

- jitter within client modem, ISP modem and buffers,

- PSTN set-up time,

- modem connection set-up time,

- ISP logon \& set-up time and

- error rate on PSTN link.

\subsection{QoS Associated with ISDN Accesses}

ISDN access uses a set bandwidth for the communication channel (16 kbit/s for the D channel, $64 \mathrm{kbit} / \mathrm{s}$ for a B channel). Aggregation of $\mathrm{n}^{*} \mathrm{~B}$ channels to provide a 384 $\mathrm{kbit} / \mathrm{s}$ channel provides a means of using video codecs even with normal RTP/UDP/TCP/IP overheads. The factors affecting QoS in this scenario are the:

- use of PPP/IP/UDP/RTP header compression on access link,

- throughput delay in ISDN terminal adapter and at ISP site,

- jitter within ISDN terminal adapter and ISP network interface buffers,

- ISDN set-up time and

- ISP Logon and session set-up time.

\subsection{QoS Issues Associated with xDSL Accesses}

xDSL access allows the use of various sizes of bandwidth, up to tens of $\mathrm{Mbit} / \mathrm{s}$, depending on application and the DSL technique used (e.g. ADSL, VDSL). IP access may use in general a mediation transport layer (i.e. ATM) or may be mapped directly into the xDSL frame (not standardized yet). The factors affecting QoS in this scenario are the:

- xDSL modem available bit rate (due to line condition and specific application),

- use of PPP/IP/UDP/RTP Header Compression on access link,

- throughput delay in xDSL modem (fast or interleaved) at ISP site,

- jitter within client modem, ISP modem and adaptation buffers,

- xDSL set-up time (e.g. when using Dynamic Power Save in VDSL application),

- ISP Logon and session set up time and

- error rate on access link.

\section{Typical QoS Measurement Campaigns}

\subsection{Intrusive Measurement Tool $=$ QoS Simulation Platform}

The main functions of the requested tool are :

- capture and analysis of the received traffic at ATM, IP and application level, taking into account the different network architectures (IPoATM, LANE/MPOA, MPLS, etc.) and their characteristics,

- performance measurement at different network layers, focusing on relevant metrics such as: throughput, one-way delay, delay variation, packet loss, etc as referred in the RFCs 1944 and 2330, 
- objective QoS evaluation, by taking into account the relevant performance parameters and network characteristics (e.g. scheduling techniques like WFQ or CBQ),

- generation of calibrated multimedia traffic patterns made of RSVP messages,

- traffic allocation to QoS Service Classes and CoS contract verification of IP streams based on Tspec parameters using a token bucket algorithm as referred in the RFC 2215. This verification would allow these streams to fit a DiffServ or IntServ network requirements,

- comparison with subjective data relating to the quality as seen by the end-users.

Iterative experiments will allow the tool to increase its knowledge in mapping objective and subjective QoS, to be able to allocate automatically the received traffic to the corresponding Service Class, only by measuring the adequate network performance. The simulation tool will enhance its QoS evaluation process by implementing innovative self-learning techniques.

\subsection{Non- intrusive Measurement Tool $=$ QoS Monitoring System}

The monitoring tool (QoS Probes + Supervision System) will have following functions :

- processing the self-learning algorithms preliminary established by using the Simulation Tool in similar network environment,

- accessing the network in real-time, without disturbing the current traffic,

- traffic allocation to QoS Service Classes,

- results supervision via data collection by a supervision system, which will be designed to be connected, via TMN interfaces, to the network administration system,

- supervision system design taking into account entities from DiffServ architecture like Bandwidth Brokers (BB) or protocols like Common Open Policy Service (COPS).

\subsection{End-to-End QoS Experiments}

Experimental services will be based on multimedia applications. Voice over IP quality will be determined according to G.711 64kB/S characteristics. Video over IP will refer to H.320 ISDN video-conferencing running at $384 \mathrm{~KB} / \mathrm{S}$. QoS expertise will take into account most types of coding and compression algorithms (G.723, H.323, M/J-PEG.).

Performance will be measured at standardized access points :

- PCM n*64KB/S,

- ISDN, basic and primary accesses,

- PDH, SDH (STM1-STM4),

- xDSL subscriber loop accesses (probably ADSL),

- Cable-modem accesses,

- ATM (PDH 34MB/S, SDH STM1-STM4). 
The evaluation process will make use of self-learning techniques, allowing statistical approach of QoS parameters. QoS expertise will take into account real-time enhancements of transport protocols (UDP/IP, RSVP, RTP, RTCP, IPV6...). As QoS management involves all terminal and network related aspects, measurement and monitoring will cover most of them. These include Tspec definition, Adspec definition if RSVP is to be used, RSVP/ATM issues, DiffServ/IntServ interface issues, DiffServ/ATM issues, MPOA/LANE QoS if used, IPv6/ATM, etc... Measurement techniques will be enhanced in order to anticipate performance degradation and to predict QoS evolution [2], [3].

Performance evaluation will be characterized by following aspects:

- connections opening and closing mechanisms,

- data transfer mechanisms,

- addressing, parameters negotiation,

- congestion and errors control,

- bandwidth allocation,

- CoS contract verification.

\section{Voice over IP Experiments}

\subsection{ETSI TIPHON Project Simulation Experiments}

The ETSI TIPHON contribution 09TD42 presented a proposal to carry out simulations where different scenarios of end to end speech transmission over IP based networks could be evaluated with respect to speech transmission quality.

In autumn 1998, an investigation according to 09TD42 was performed by Deutsche Telekom Berkom (T-Berkom) where such different scenarios were simulated and subjectively assessed in a well-established listening. The simulation processing contained a couple of speech codecs, packet loss ratios and various kinds of audio frames per IP packet. This TD describes the test methodology, the simulation method, the scenarios and the results of the executed simulation processing.

Furthermore we would like to discuss some interesting results concerning the relationship of speech material (construction, length) packet loss and their influence to the auditory assessment.

\subsubsection{Test Methodology}

TIPHON WG5 (DTR/TIPHON 05001 V1.2.5, chapter 7.3.2) defined a methodology for testing speech quality in TIPHON compliant networks and terminals. This methodology was taken into account and used as a basis model for the $\mathrm{T}$ Berkom simulation processing.

A set of speech signals designed according to ITU-T Rec. P.800 was used as input of the simulation path. The simulation path includes the terminal side (electrical part) and the network itself. The influence of the terminal side was focussed to the speech 
conversion and IP packet size issue. The influence of the network side was simulated by different packet loss rates. After the simulation the speech samples were recorded and stored in a database.

\subsubsection{Simulation Method}

For simulation of network influences in the case of packet loss, a common channel model was designed, realized by channel files which describe the network condition with the same time resolution as the source speech sample rate. So the network has a certain condition (good or bad) for every speech sample (every $125 \mu$ s) two adjacent network states were considered as statistically independent, because the network speed was assumed to be much higher than the sample rate (8000 samples per second). So for each packet loss rate one channel file was created using a random generator. The length of this channel file was exactly the same as the length of the speech file.

In a further step the speech file, assembled in IP packets, was matched to the channel file. According to the length of the IP packet $(10 \mathrm{~ms}, 20 \mathrm{~ms}, \ldots)$ the channel file was checked every time when a packet was ready to send. That means if the packet size was $10 \mathrm{~ms}$ the channel file was checked also every $10 \mathrm{~ms}$ if the condition is good or bad. In a bad case the IP packet was lost, otherwise it was further processed. This information (IP packet lost or not) was stored in a description file which was the input of the re-assembler and speech decoder.

\subsection{Testing of Speech Quality}

There are two methods of testing end-to-end (acoustic to acoustic) speech quality:

- subjective tests involving the opinion of panels of users (see ITU-T Recommendation P.800),

- objective tests including comparison methods against a known reference signal (See ITU-T Recommendation P.861), absolute estimation methods.

Based on ITU-T Recommendation P.561, the measurement of individual parameters followed by the use of a Transmission Rating Model (TRM) to combine the effects of the individual parameters and predict the subjective views of users. The E-model is under consideration for this purpose.

Subjective tests have the advantage of including all parameters and providing a direct subjective view, but they take a long time to perform, are costly and are ill-suited to investigating changes in the values of many parameters because of the large numbers of combinations involved.

Objective comparison methods are described in DEG/STQ00001. Objective tests using the E-Model approach should include the same parameters as in the PSTN world: 
- SLR Sending Loudness Rating;

- RLR Receiving Loudness Rating;

- OLR Overall Loudness Rating;

- STMR Sidetone Masking Rating;

- LSTR Listener Sidetone Rating;

- Ds D-Value of Telephone at Send-side;

- Dr D-Value of Telephone at Receive-side;

- WEPL Weighted Echo Path Loss;

- qdu Number of Quantizing Distortion Units;

- Ie $\quad$ Equipment Impairment Factor (low bit-rate Codecs);

- Nc Circuit Noise referred to the $0 \mathrm{dBr}$-point;

- Nfor Noise Floor at the Receive-side;

- Ps Room Noise at the Send-side;

- Pr Room Noise at the Receive-side.

For evaluation of the Ie values for low bit-rate codecs, some objective measurement methods have been developed but commercial measurement systems are not yet available. In addition, specific requirements from the TIPHON system (eg. packet loss) have to be considered in determining Ie.

In conversational situations:

- TELR Talker Echo Loudness Rating;

- T Mean one way delay of the echo path; and

- Tr Roundtrip Delay in a closed 4-wire loop,

need also to be considered.

The performance of TIPHON systems in terms of TIPHON speech quality classes may also be measured between the electrical input/outputs of the TIPHON terminals or SCN telephone terminals connected to the TIPHON system. Figure 2 shows in general how this should be done.

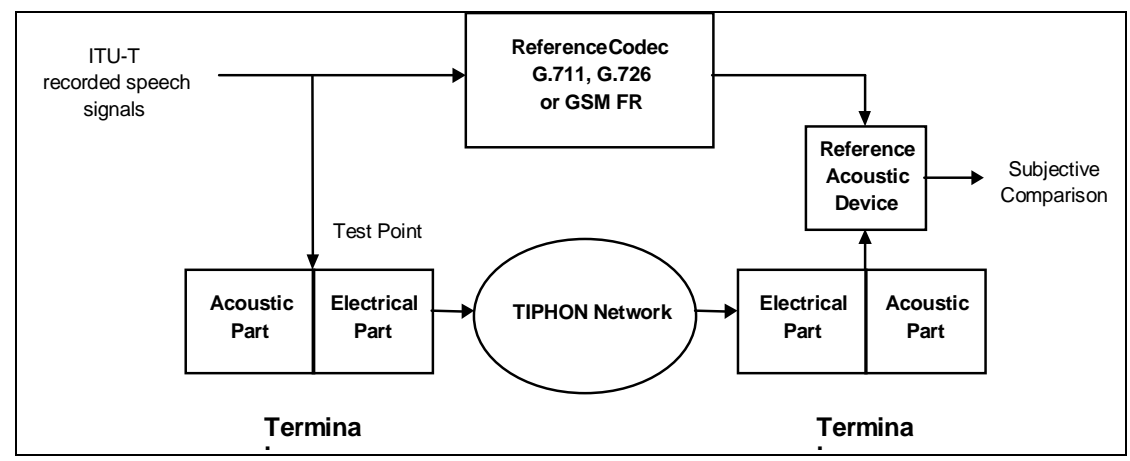

Fig. 2: Methodology for testing TIPHON speech quality

Speech quality shall be measured using the subjective test methodology as defined by ITU-T SG12 until such times as calibrated objective methods are possible. It is 
planned that these test results will be used in the future to enable predictions of overall performance to be made using a TRM (e.g. the E-Model). It should be noted that the E-model is not a test method.

\section{Desktop Videoconferencing Experiments}

\subsection{State of the Art}

\subsubsection{State of the Art of QoS in IP Infrastructures}

For many years, public network operators regarded ATM as the solution for a service integrating broadband network. Conceived as a logical extension of narrowband ISDN, its standardization was influenced by the connection-oriented paradigm, signaling protocols and addressing scheme known from ISDN. While ATM research, development and standardization was concerned with guaranteed QoS for typical broadband ISDN applications, such as video-on-demand and multimedia conferencing as well as with an efficient rate control for data applications (Available Bit Rate), the World Wide Web helped to establish IP networks as the carrier for data networking.

The current Internet architecture offers a flexible, but simple connectionless best effort service and this is inadequate for applications sensitive to the QoS provided by the network. For this reason, the IETF has been working on extensions to the current Internet protocol suite in order to enable service guarantees (Resource Reservation Protocol RSVP together with Integrated Services, IIS) or at least differentiation (Differentiated Services, DS).

In Integrated Services, RSVP allows applications to request either Guaranteed or Controlled Load Service for individual flows in an IP network. However, the wide scale deployment of RSVP must be approached with care because the processing of (periodically refreshed) reservation and control messages, the identification of each packet based on the IP header and the handling of per-flow reservation state becomes challenging in backbone routers passed by a huge number of individual flows.

Several ACTS projects (DIANA, SUSIE, BTI, PETERPAN, IthACI and ELISA) have been working on the implementation and optimization of a RSVP over ATM control architecture to integrate IP and ATM while enforcing QoS end-to-end. Their work extends standardized solutions to integrate IP and ATM on a best-effort basis, namely Classical IP over ATM (CLIP), Next Hop Resolution Protocol (NHRP), Multicast Address Resolution Server (MARS), LAN Emulation (LANE) and Multiprotocol over ATM (MPOA) that focus on IP to ATM address resolution to set-up switched best-effort ATM VCs.

The resulting RSVP over ATM architecture is an example of traffic descriptor and QoS parameter based resource reservation that guarantees tight QoS end-to-end. The 
aforementioned scalability issues are addressed by applying a concept of massive aggregation of flows to a single VC.

In contrast, Differentiated Services architecture achieve scalability by classifying and marking packets by means of the so-called DS field in the IP header once at the ingress to a DS capable IP network. Based on a (relatively static) Service Level Agreement profile negotiated between an Internet provider and an user, traffic will receive a particular per-hop forwarding behavior on routers that interpret the DS field. Without explicit signaling and admission control, DS have the potential to provide relative QoS to so-called behavior aggregates (streams that are marked with the same priority) using simple and scalable mechanisms.

Hence, the Internet is evolving in the direction of a multi-service network that supports several traffic classes, signaling and various other attributes associated with a stream of data. Since various solution will co-exist, QoS applications and services have to build upon a more generic protocol layer, as represented by the H.323 series of recommendations, that is to be translated to native network layers and traffic control capabilities in terminals (with operating systems support) or gateways (network equipment vendors). This approach enables application developers to keep pace with and to make use of the rapidly evolving IP based technologies and network infrastructures.

\subsubsection{State of the Art in QoS Evaluation Methodologies, Tools, and Experiments}

Today, many of activities address QoS evaluation methods and tools dedicated to broadband networks; most of them concentrate on objective (quantifiable, measurable) QoS aspects, which are mainly network oriented. Subjective aspects relate to the user's point of view and are only approached qualitatively.

\section{Objective QoS}

The IETF's work on Quality of Service and performance is primary directed towards developing new protocols which will allow a degree of bandwidth reservation or Quality of Service differentiation. The IP Performance Metrics working group (IPPM) is working on defining a set of standard metrics that can be used to derive a quantitative measure of the quality, performance and reliability of Internet data delivery services.

Measurement and monitoring processes will also take into account work performed in :

- ACTS Project ISABEL: video-conferencing traffic characteristics, ISABEL and Mbone tools,

- Project MEHARI (Spanish local project): techniques and tools for the analysis of Internet services. Currently the MEHARI system is used to do measurements on IP over ATM. New functionality could be as for example LANE/MPOA measurements, end-to-end QoS monitoring, etc. 
- Project SABA (Spanish local project): new services and protocols for the Broadband Spanish Academic Network. It is a project on next generation Internet

(QoS management, terminal and network related aspects).

\section{Subjective QoS}

QoS requirements by the user/customer is the statement of the level of quality of a particular service required or preferred by the user/customer. The level of quality may be expressed by the user/customer in technical or non-technical language.

A typical user/customer is not concerned with how a particular service is provided or with any of the aspects of the network's internal design, but only with the resulting end-to-end service quality. From the user's/customer's point of view, QoS is expressed by parameters that:

- focus on user/customer-perceivable effects, rather than their causes within the network,

- do not depend in their definition on assumptions about the internal design of the network,

- take into account all aspects of the service from the user's/customer's point of view,

- may be assured to a user/customer by the service provider(s),

- are described in network independent terms and create a common language understandable by both the user/customer and the service provider.

\section{Classes of Service}

The terminology Class of Service is used to describe a scheme where service types are grouped logically together. This grouping can then be used as the basis for service type prioritization.

Four classes of service are defined by ETSI:

- Class4 : Best quality,

- Class3 : High Quality,

- Class2 : Medium Quality,

- Class1 : Best Effort Quality.

Different (and complementary) approaches are being defined in the two main QoS architectures of the IETF [5]. These architectures will be prevalent in the near future Internet:

- Differentiated Services (DiffServ):

. Default (DE): best-effort,

. Assured forwarding (AF): not completed yet,

. Expedited forwarding (EF): not completed yet.

- Integrated Services (IntServ):

. Guaranteed: with bandwidth, bounded delay and no-loss guarantees,

. Controlled load: simulates a best-effort service in a lightly loaded network,

. Best-effort. 
One important issue under work is the mapping of CoS definitions of both standards IntServ and DiffServ.

\subsubsection{State of the Art of Multimedia Conferencing Systems}

Almost all multimedia or video conferencing systems used today are either based on IP (e.g. CUSeeMe, the MBone tools and Microsoft's Netmeeting) or on ISDN (e.g. Intel ProShare and PictureTel). This leads to a conservative dimensioning where the application on the one hand must be able to deal with data losses and on the other hand must put restrictions on its output bit rate to protect the other services running over the same network from excessive losses.

This scheme works very well for data, but is problematic for real-time audiovisual services. Furthermore the available bit-rate on the Internet is quite low which leads to designs with low resolution (image size) and low frame-rates. With the use of ATM these problems could be reduced or eliminated.

Multimedia conferencing systems using native ATM are not commercial available today, but some research projects have worked in this area. The RACE project R2025 MIMIS have worked on multimedia desktop conferencing for ATM networks, using the early drafts of the ITU-T T.120 series.

\subsection{The DIVINE Project}

DIVINE is an European consortium regrouping industrial companies, research centers, telecommunications operators, universities and end-users [4]. The DIVINE project was initiated by the European Commission (DGXIII) in the frame of the $4^{\text {th }}$ PC\&RD ACTS Program.

The main objectives of the DIVINE project were:

- to be a "market-driven" project through operational field trials involving real end users, to issue meaningful conclusions on the viability of the deployment of multipoint multimedia applications on high speed communication infrastructures,

- to be a "product-oriented" project : the reuse of the results of the project is a key issue. It implies that the DIVINE system has to fit with the user requirements related with functionality, quality of service and price,

- to demonstrate the interoperability between the B-ISDN DIVINE and N-ISDN commercial videoconferencing products,

- to demonstrate on a large scale experimentation the interoperability of European ATM-LAN with the ATM-WAN,

- to promote the standards in broadband desktop videoconferencing applications and to contribute to the standardization work in IMTC, ETSI or ITU-TS [6].

Bandwidth-on-demand should be easily and flexibly controlled by the user. Support of a variety of interface-cards for ATM, graphics, video-input and video compression should be integrated. Access to these peripheral components should be enabled via 
hardware interfaces. If such an interface does not exist, at least a standardization should be considered or this should be open to the public.

The DIVINE protocol stacks can be described as follows:

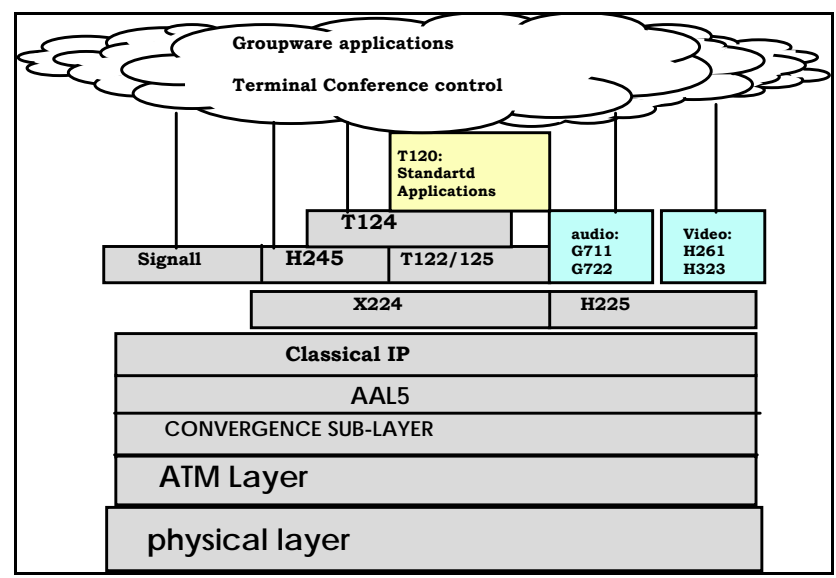

Fig 3: The DIVINE protocol stack

Unicast and multicast videoconferencing is requested, as well as easy interworking with ISDN based videoconferencing facilities.

\subsection{Tests and Performance Measurements}

The host configuration includes machines, graphic interfaces, audio/video codecs and the network access interfaces includes LAN Ethernet: $10 \mathrm{Mbit} / \mathrm{s}, 100 \mathrm{Mbit} / \mathrm{s}$, Fast Ethernet, Switched Ethernet, $155 \mathrm{Mbit} / \mathrm{s}$ ATM. Quality of Service evaluation relates to end-to-end multimedia applications with LAN-to-LAN interconnection, audio/video conferencing and audio/video transmission and distribution.

The main test functions are the user/network protocol monitoring, the user/network protocol simulation, the traffic load simulation and the errors insertion (bit/cell/frame errors, delays, jitter...).

In user simulation mode, the ATM analyzer acts as any number of user devices which may communicate with a real ATM switch under test. A real ATM switch is attached to the analyzer which may simulate any number of user devices which are called virtual stations. Additionally, the analyzer enables the simulation of any number of user devices which may communicate with the simulated switch or with any other user device. The measurements include:

- estimation of bandwidth allocated to a videoconferencing session,

- videoconferencing behavior under strong network load conditions,

- audio/video quality estimation,

- determination of optimal Quality/Bitrate relations, 
- interoperability with ISDN (H320/H323 gateway tests),

- native ATM interworking (ATM access for DIVINE terminal).

Objective measurements were done essentially by observing traffic at the ATM level (Radcom monitoring equipment) and at the Ethernet level (Meterware monitoring equipment), via cable-modems and via ISDN H320 gateway [15].

During our tests, both videoconferencing applications are adjusted for maximum video quality, (the maximum bandwidth allocated for DIVINE is $750 \mathrm{kbit} / \mathrm{s}$ ). Statistics are extracted with the RADCOM test equipment, connected to the ATM network and the results are depicted for a 1 minute connection (data storage capabilities).

The results can be represented in this following figure:

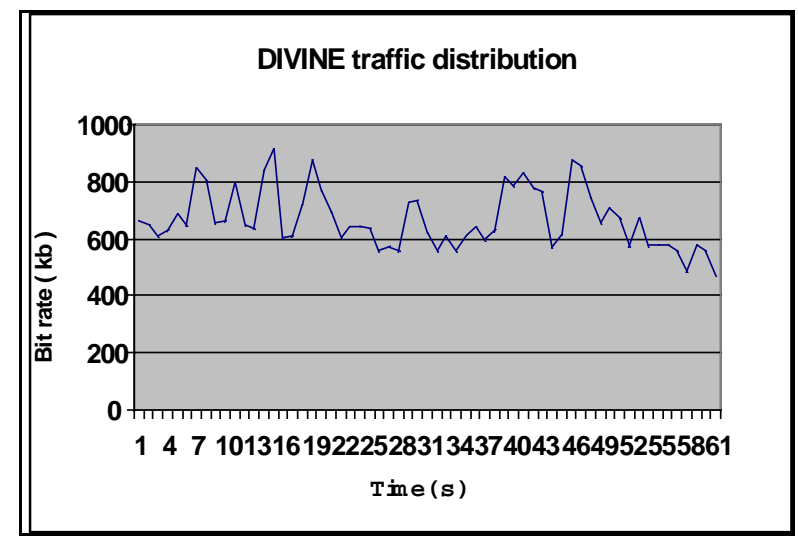

Fig. 4 : DIVINE traffic distribution

Decreasing available bandwidth affects video and audio quality, but the connections are not lost. The maximum quality requests only $450 \mathrm{kbit} / \mathrm{s}$ to $900 \mathrm{kbit} / \mathrm{s}$ bandwidth allocation and videoconferencing applications request a minimum of available bandwidth to provide acceptable video and audio quality.

Fugitive traffic congestion disturbs the video transmission for short periods of time, but does not affect the videoconferencing session itself. When the available bandwidth increases again, video and audio quality recover their initial values.

\section{The Future of VoIP Networks}

New operators use more and more VoIP because they can use the same equipment to transmit voice and data over Internet. The development of VoIP implies the integration of the PSTN (Public Switched Telephone Network) network. Therefore gateways that can be used to interconnect SS7 protocol with IP protocol are now available. The three majors protocols for VoIP and SS7 over IP are H.323, MGCP (Media Gateway Control Protocol) and SIP (Session Initiative Protocol). 


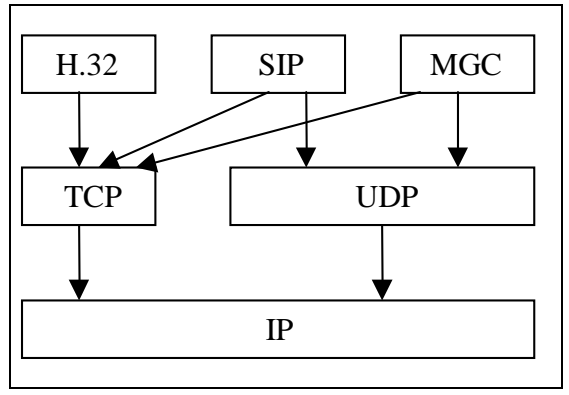

Fig. 5: VoIP and SS7 over IP protocols

The H.323 ITU (International Telecommunication Union) standard is adapted for multimedia conferences. It can be used to transmit voice over ATM, but H.323 cannot really evolve to integrate the SS7 signalization.

The MGCP IETF (Internet Engineering Task Force) standard, which come from the merge of SGCP (Simple Gateway Control Protocol) with IPDC (Internet Protocol Device Control), has been developed to resolve the SS7/VoIP integration. Then, MGCP can be used to offer operational VoIP networks based on PSTN over IP.

The SIP IETF standard has been initially developed for the multimedia communication over the Mbone (Internet Multicast Backbone). SIP offers SS7 over IP mechanisms and can be used instead of H.323. SIP is simpler than MGCP that offer more control mechanisms.

In the future, it will be important to evaluate how SIP or MGCP can be managed by the DiffServ and MPLS protocols.

\section{Conclusion}

The QoS for VoIP systems and desktop videoconferencing is more and more enhanced. In this article, we present some methods and performance measurements applied to VoIP and to desktop videoconferencing systems. Some methods for the simulations and for the tests are experimented in different projects.

\section{References}

1. K. Achtmann, K.H. Doring, R. Herber, G. Komp, “An ATM-based demonstration model for multimedia services using different access networks", Multimedia Applications, Services and Technologies, ECMAST'97, May 21-23, 1997, Milan, Italy, 1-17.

2. G. Armitage, "MPLS: the Magic behind the Myths", IEEE Communications Magazine, Vol. 38, No 1, , January 2000, 124-131.

3. R. Cocca, S. Salsano, M. Listanti, "Internet Integrated Service over ATM: a Solution for Shortcut QoS Virtual Channels, IEEE Communications Magazine, Vol. 37, No 12, , December 1999, 98-104. 
4. DIVINE (Deployment of Interpersonal Videoconferencing Systems on IBC Networks) project, ACTS European Project AC035, 1998.

5. G. Eichler, H. Hussmann, G. Mamais, I. Venieris, C. Prehofer, S. Salsano, "Implementing Integrated and Differentiated Services for the Internet with ATM Networks: a practical approach", IEEE Communications Magazine, Vol. 38, No 1, January 2000, 132-141.

6. ETSI/DTR/TIPHON-05001 V1.2.5., "Telecommunications and Internet Protocol Harmonization Over Networks ; general aspects of Quality of Service", 1998

7. B. Goodman, "Internet Telephony and Modem Delay", IEEE Network, Vol. 13, May-June 1999, 8-16.

8. A.M. Grilo, P.M. Carvalho, L.M. Medeiros, M.S. Nunes, "VTOA/VoIP/ISDN Telephony Gateway", 2nd International Conference on ATM, ICATM'99, Colmar, June 21-23 1999, 203-235.

9. ISO/IEC DIS13236 , "Information Technology Quality of Service - Framework," 1996.

10. N. Kroth, L. Mark, J. Tiemann, " A Framework for Testing IP QoS over ATM Networks: Implementation and Practical Experiences", 2nd International Conference on ATM, ICATM'99, Colmar, June 21-23 1999, 203-235.

11. V. Mirchandani, D. Everitt, "Performance of integrated telephony service over standalone LAN and ATM interworked LANs", $22^{\text {nd }}$ Conference on Local Computer Networks, Minneapolis, USA, November, 2-5, 1997, 80-88.

12. D. Newman, "VoIP Gateway: voicing doubts", Data Communications International, Vol. 38, No 12, September 1999, 70-78.

13. Z. Peifang, "Scalability and QoS guarantee in IP networks", $8^{\text {th }}$ International Conference on Computer Communications and Networks, 627-633.

14. X. Scharff, P. Lorenz, "Specification of a Multimedia Application Generator in Telecommunication Systems", $12^{\text {th }}$ International Conference on Computer Applications in Industry and Engineering (CAINE'99), Atlanta, USA, November 4-6 1999, 54-57.

15. H. Tobiet, P. Lorenz, "Performance measurements on an ATM-based Metropolitan Area Network: OASICE Case Study", 1st IEEE International Conference on ATM, ICATM'98, Colmar, June 22-24 1998, France, 410-417.

16. J. Toga, J. Oltt, "ITU-T Standardization Activities for Interactive Multimedia Communications on Packet-based Networks: H.323 and related recommendations", Computer Networks, Vol. 31, No 3, February 199,9 205-223.

17. M. Valino, J. Corchado, "VoIP: the convergence of Networks", Computing and Imformation Systems, Vol. 6, No 3, October 1999, 105-112.

18. P.P. White, "ATM switching and IP Routing Integration: the Next Stage in Internet Evolution", IEEE Communication Magazine, April 1998, 78-83. 